\title{
Challenges in Diagnosing Vaping-Associated Acute Lung Injury During the COVID-19 Pandemic
}

\author{
Montreh Tavakkoli ${ }^{1}$, Nabeel Wahid ${ }^{1}$, Carly Borinsky ${ }^{1}$, Dennis Toy $^{1}$, and Tanping Wong ${ }^{1}$ \\ ${ }^{1}$ NewYork-Presbyterian Hospital/Weill Cornell Medical Center
}

June 24, 2020

\begin{abstract}
We encountered a 34-year-old man with a significant vaping history who presented with acute hypoxic respiratory failure, headaches and gastrointestinal symptoms during the COVID-19 pandemic. Based on his laboratory and chest CT findings, we had a high clinical suspicion for COVID-19 and EVALI, yet distinguishing the diagnoses proved challenging. His SARS-CoV-2 nasopharyngeal PCR swabs were negative. However, this test has a low sensitivity. COVID-19 serology testing was negative, and the patient was ultimately diagnosed with EVALI based on subtle chest CT findings. To our knowledge, this is the first paper highlighting the similarities and distinguishing features of EVALI and COVID-19 acute lung injury.
\end{abstract}

To the Editor:

Given the high prevalence of coronavirus disease 2019 (COVID-19), it can be difficult to identify alternative causes of acute lung injury, including e-cigarette or vaping product use-associated lung injury (EVALI). We encountered a 34-year-old man with a significant vaping history who presented with acute hypoxic respiratory failure, headaches and gastrointestinal symptoms during the COVID-19 pandemic. Based on his laboratory and chest CT findings, we had a high clinical suspicion for COVID-19 and EVALI, yet distinguishing the diagnoses proved challenging. His SARS-CoV-2 nasopharyngeal PCR swabs were negative. However, this test has been shown to have a low sensitivity of $63-79 \%^{1,2}$. COVID-19 serology testing was negative, and the patient was ultimately diagnosed with EVALI based on subtle chest CT findings. To our knowledge, this is the first paper highlighting the similarities and distinguishing features of EVALI and COVID-19 acute lung injury.

Common features of EVALI and COVID-19 include presentation at a median of 5-6 days following onset of constitutional, respiratory and/or gastrointestinal symptoms; exam findings of fevers, tachycardia, tachypnea and hypoxia; as well as laboratory evidence of mild transaminitis and elevated inflammatory markers ${ }^{3,4,5}$. Weight loss and chest pain are commonly observed in EVALI ${ }^{3}$. Anecdotally, we have also witnessed significant weight loss due to anosmia and loss in appetite as well as fatigue, chest pain and headaches among patients with COVID-19. While COVID-19 more commonly presents with lymphopenia, thrombocytopenia and acute kidney injury, EVALI is more likely to present with abdominal pain, leukocytosis, hyponatremia, hypokalemia and elevated procalcitonin. However, these features are also observed in patients with COVID-19 (Table $1)^{3,4,6}$.

Despite this, subtle differences in chest imaging can help differentiate EVALI from COVID-19. The most common findings of EVALI on chest CT include bibasilar-predominant consolidations and ground-glass opacities, often with a centilobular distribution and lobular or subpleural sparing ${ }^{3,6,7}$. In contrast, COVID-19 commonly presents with bilateral, peripheral and basilar predominant ground-glass opacities or consolidations that have extensive geographic distributions ${ }^{8}$. In contrast to EVALI, centrilobular ground glass nodules 
are considered atypical for COVID- $19^{8}$.

Clinical outcomes include greater rates of admission to the intensive care unit and invasive mechanical ventilation in EVALI despite longer lengths of stay and higher mortality in COVID-19. Mortality among those [?]53y.o. is $2 \%$ relative to a $7 \%$ among patients $<50$ y.o. for EVALI and COVID- $19^{3,5}$. In line with these findings, our patient was admitted to the intensive care unit and was discharged by day 8 of admission.

Collectively, EVALI and COVID-19 should be included in the differential diagnosis of any patient presenting with constitutional, respiratory and/or gastrointestinal symptoms who has vaped within the last 90 days. While clinical and laboratory findings may not be able to accurately delineate EVALI from COVID-19 and chest CT imaging can substantially overlap, certain patterns such as centrilobular ground glass nodules and subpleural sparing are more commonly found in EVALI. These subtle differences can aid in making the appropriate diagnosis.

\section{References}

1. He JL, et al. Diagnostic performance between CT and initial real-time RT-PCR for clinically suspected 2019 coronavirus disease (COVID-19) patients outside Wuhan, China. Respir Med . 2020;168:105980.

2. Wang W, et al. Detection of SARS-COV-2 in Different Types of Clinical Specimens. JAMA . 2020;323(18):1843-1844.

3. Layden JE, et al. Pulmonary Illness Related to E-Cigarette Use in Illinois and Wisconsin - Final Report. NEJM . 2020;382:903-916.

4. Goyal P, et al. Clinical Characteristics of Covid-19 in New York City.NEJM . 2020;c2010419.

5. Richardson S, et al. Presenting Characteristics, Comorbidities, and Outcomes Among 5700 Patients Hospitalized With COVID-19 in the New York City Area. JAMA . 2020;323(20):2052-2059.

6. Huang C, et al. Clinical features of patients infected with 2019 novel coronavirus in Wuhan, China. The Lancet . 2020;395:497-506.

7. Henry TS, et al. Imaging of Vaping-Associated Lung Disease. NEJM . 2019;381:1486-1487.

8. Simpson S, et al. Radiological Society of North America Expert Consensus Statement on Reporting Chest CT Findings Related to COVID-19. Endorsed by the Society of Thoracic Radiology, the American College of Radiology, and RSNA. J Thorac Imaging . 2020;10.1097/RTI.0000000000000524.

\section{Hosted file}

Table 1.docx available at https://authorea.com/users/336478/articles/462136-challenges-indiagnosing-vaping-associated-acute-lung-injury-during-the-covid-19-pandemic 